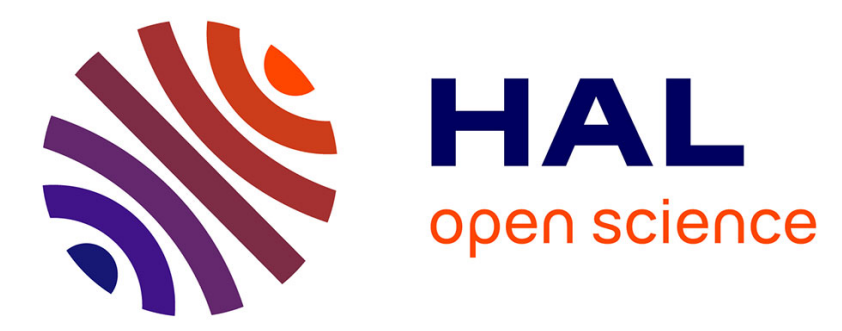

\title{
Generating Soap Bubbles by Blowing on Soap Films
}

\author{
Louis Salkin, Alexandre Schmit, Pascal Panizza, Laurent Courbin
}

\section{To cite this version:}

Louis Salkin, Alexandre Schmit, Pascal Panizza, Laurent Courbin. Generating Soap Bubbles by Blowing on Soap Films. Physical Review Letters, 2016, 116 (7), pp.077801. 10.1103/PhysRevLett.116.077801 . hal-01277957

\section{HAL Id: hal-01277957 \\ https://hal.science/hal-01277957}

Submitted on 23 Feb 2016

HAL is a multi-disciplinary open access archive for the deposit and dissemination of scientific research documents, whether they are published or not. The documents may come from teaching and research institutions in France or abroad, or from public or private research centers.
L'archive ouverte pluridisciplinaire HAL, est destinée au dépôt et à la diffusion de documents scientifiques de niveau recherche, publiés ou non, émanant des établissements d'enseignement et de recherche français ou étrangers, des laboratoires publics ou privés. 


\title{
Generating soap bubbles by blowing on soap films
}

\author{
Louis Salkin, Alexandre Schmit, Pascal Panizza, ${ }^{*}$ and Laurent Courbin ${ }^{\dagger}$ \\ IPR, UMR CNRS 6251, Campus Beaulieu, Université Rennes 1, 35042 Rennes, France
}

\begin{abstract}
Making soap bubbles by blowing air on a soap film is an enjoyable activity, yet a poorly understood phenomenon. Working either with circular bubble wands or long-lived vertical soap films having an adjustable steady state thickness, we investigate the formation of such bubbles when a gas is blown through a nozzle onto a film. We vary film size, nozzle radius, space between the film and nozzle, and gas density, and we measure the gas velocity threshold above which bubbles are formed. The response is sensitive to containment, i.e., the ratio between film and jet sizes, and dissipation in the turbulent gas jet which is a function of the distance from the film to the nozzle. We rationalize the observed four different regimes by comparing the dynamic pressure exerted by the jet on the film and the Laplace pressure needed to create the curved surface of a bubble. This simple model allows us to account for the interplay between hydrodynamic, physicochemical, and geometrical factors.
\end{abstract}

PACS numbers: 47.55.N- 47.55.Ca 47.55.db

Thin liquid films are widespread in nature and industry [1]. Interesting for both practical and theoretical reasons, these fluid systems are very popular and they are a major source of entertainment when they come in the form of soap bubbles. Blowing soap bubbles with a child's wand is indeed an activity that can be fascinating for people at any age. Popular in paintings through the ages [2] and useful to illustrate scientific principles in a classroom setting, soap bubbles have been studied scientifically for centuries [3-5]. These soap-film-based structures truly impact a wide swath of phenomena in fields ranging from foam science [6] to cosmology [7]. Blowing soap bubbles can even inspire new ways to create lipid vesicles [8] and be beneficial to pursed-lip breathing techniques [9]. Despite the immense use of bubbles for either entertainment, scientific, or educational purposes, understanding how bubbles form when air is blown onto a soap film, as illustrated in Fig. 1(a), has surprisingly remained a briefly mentioned, yet unaddressed, issue [4, 10].

To answer this question, we investigate the impact dynamics of a gas jet with a soap film. Inspired by works on two-dimensional turbulence [11-13], our setup revolves around a vertically falling soap film [see Fig. 1(b)]. Unlike common bubble wands [Fig. 1(a)], this setup allows us to work with long lived films that have adjustable, uniform, and steady state thicknesses. These kinds of giant soap films have also been used to study the impact and subsequent tunneling of liquid drops [14]. Not only gases but also liquid drops and jets and solid spheres can indeed pass through a soap film without popping it [1418]. We also work with circular bubble wands made of wire for comparison purposes. Varying gas density and velocity, film size, radius of the nozzle emitting the jet, and distance between film and nozzle, our experiments show that bubbles form only when the gas speed exceeds a threshold (Fig. 1 defines parameters at play and primary experimental outcomes). Working with vertical soap films having a stationary thickness allows us to show that, in our experiments, this threshold in velocity depends on neither the film's thickness nor its hydrodynamics. The thorough experimental investigation of this threshold unveils four possible regimes, the occurrence of which depends on the degree of containment defined as the ratio between film and jet sizes and dissipation in the jet which is a function of the gap between nozzle and film. In each case, we rationalize experimental findings with a simple model based on a comparison between the dynamic pressure of the jet on the film and the Laplace pressure needed to create the curved surface of bubbles.
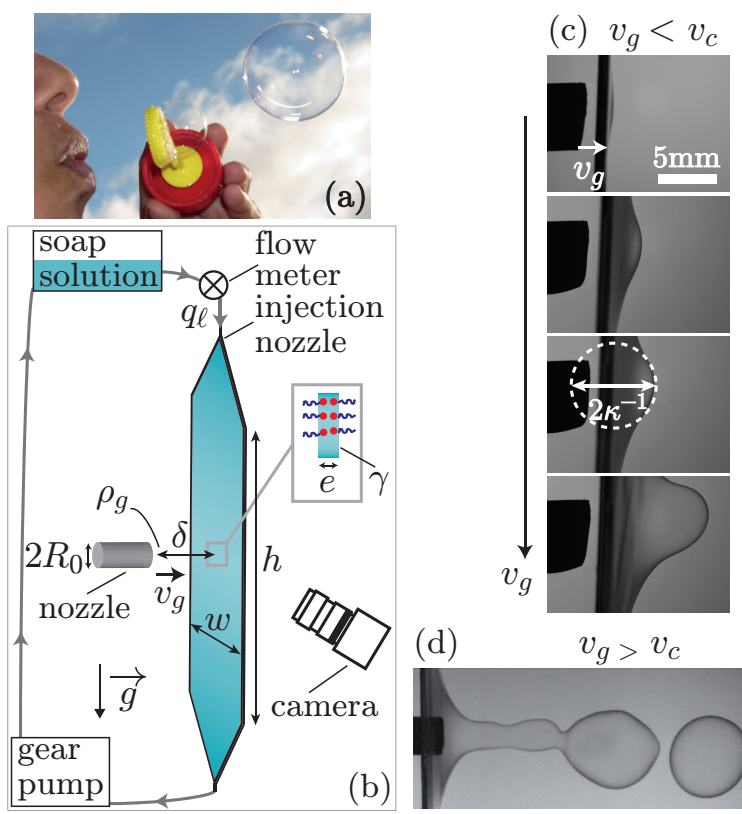

(d)

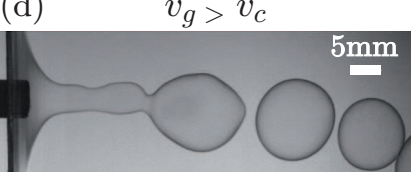

FIG. 1. (color online) (a) Photograph illustrating soap bubble blowing with a commercial bubble wand. (b) Schematic of the experimental setup defining geometric $\left[R_{0}, \delta, w, e, h\right]$, physicochemical $\left[\gamma, \rho_{g}\right]$, and hydrodynamic $\left[q_{\ell}, v_{g}\right]$ variables. (c) Typical evolution of the cavity (radius of curvature $\kappa^{-1}$ ) formed in a film as the gas speed $v_{g}$ increases and is smaller than the threshold $v_{c}$. (d) Bubbles are created for gas speeds larger than $v_{c}$. 
We generate self-sustained soap films with the setup depicted in Fig. 1(b). A tank placed above this setup contains a solution for blowing bubbles (Potentier) which flows under gravity to form a soap film flowing between two nylon wires at a flow rate $q_{\ell}=10^{-9}-5 \times 10^{-8} \mathrm{~m}^{3} \mathrm{~s}^{-1}$ controlled and measured by a flow meter (AALBORG). The typical height of a film is $h \simeq 1 \mathrm{~m}$ and its width $w$ varies in the range $1-15 \mathrm{~cm}$. We measure the liquid dynamic viscosity $\eta_{\ell}=50 \mathrm{mPa}$ s and air-liquid surface tension $\gamma=24 \mathrm{mN} \mathrm{m}^{-1}$ with an Anton Paar rheometer and pendant drop tensiometry (Teclis), respectively. A spectrometer (Avantes) measures the steady state film thickness $e=1-5 \mu \mathrm{m}$ far downstream the fluid injection nozzle in a section where the film is homogeneous and the liquid has reached a terminal velocity [11]; $e$ can be tuned by changing the ratio $q_{\ell} / w[12,19]$. A gear pump (Cole-Parmer) pumps back the liquid into the tank so that it flows in a closed circuit indicated by the gray arrows in Fig. 1(b); a soap film then lasts as long as fluid is supplied. Using a flow control system (Fluigent), we inject a gas at controlled pressure through a nozzle of radius $R_{0}=10^{-4}-10^{-1} \mathrm{~m}$. When $R_{0}<10^{-2} \mathrm{~m}$, the average exit velocity of the gas $v_{g}=q_{g} / \pi R_{0}{ }^{2}=10^{-1}$ $100 \mathrm{~m} \mathrm{~s}^{-1}$ is obtained from the measurement of the gas flow rate $q_{g}=10^{-6}-10^{-4} \mathrm{~m}^{3} \mathrm{~s}^{-1}$ with a flow meter (McMillan). For the largest values of $R_{0}$, we use a wind tunnel (Leybold) to generate gas jets and hot wire anemometry (Testo) to characterize the radial profile of their axial velocity. A jet impacts a soap film at a normal incidence and a camera (Photron SA3) records side views of the flow. To vary the gas density, we use either helium (density $\left.\rho_{g}=0.17 \mathrm{~kg} \mathrm{~m}^{-3}\right)$, air $\left(\rho_{g}=1.2 \mathrm{~kg} \mathrm{~m}^{-3}\right)$, or sulfur hexafluoride $\left(\rho_{g}=6.2 \mathrm{~kg} \mathrm{~m}^{-3}\right)$. The gap $\delta$ between nozzle's orifice and film ranges from essentially zero $(\delta \simeq 0)$ to $10^{-1} \mathrm{~m}[20] . R(\delta)$ herein denotes a gas jet's radius at $\delta$; see [19] for details on the shape of a jet.

For any distance $\delta$ and degree of containment $w /[2 R(\delta)]$, our experiments show that bubbles form only when $v_{g}$ exceeds a threshold $v_{c}$ (see Fig. 1(d) and movie $\mathrm{S} 1$ in [19]). For gas speeds below $v_{c}$, the jet deforms the film and creates a cavity whose radius of curvature $\kappa^{-1}$ decreases with $v_{g}$ (Fig. 1(c) and movie S2 in [19]). For any set of variables $q_{\ell}$ and $w$, we find that $v_{c}$ does not depend on film's thickness nor hydrodynamics [19]. In what follows, we investigate the evolution of $v_{c}$ with $\delta$ for jets that are either contained $[w / 2>\boldsymbol{R}(\delta)]$ or un-contained $[\boldsymbol{w} / \mathbf{2}<\boldsymbol{R}(\delta)]$.

We begin by studying the case of contained jets with $\delta \simeq 0$ which corresponds to $R(\delta)=R_{0}$. Figure 2(a) shows the variations of $v_{c}$ with $R_{0}$ for the three gases. These results suggest that $v_{c}$ is a decreasing function of both gas density and $R_{0}$ with $v_{c} \propto 1 / \sqrt{R_{0}}$. Also shown in this figure, data obtained with vertically falling films (closed symbols) mirror measurements obtained with circular bubble wands made of wire and having a diameter $w$ (open symbols) which indicates that our setup in Fig. 1(b) is pertinent to the real-world situation, i.e., blowing bubbles with commercial wands as in Fig. 1(a). In our experiments, the Reynolds number $\mathcal{R}=$ $\frac{\rho_{g} v_{g} R_{0}}{\eta_{g}}=5 \times 10^{2}-5 \times 10^{3}$ is large; $\eta_{g}=(1.5-1.9) \times$ $10^{-5} \mathrm{~Pa} \mathrm{~s}$ is the gas dynamic viscosity. For these inertiadominated flows, we try to rationalize our experimental results by simply comparing estimates of the dynamic pressure of the jet on the film, $\frac{1}{2} \rho_{g} v_{g}{ }^{2}$, and the Laplace pressure needed to create the cavity, $4 \gamma \kappa$. Observations made at the threshold show that bubbles form when $\kappa^{-1}$ is comparable to $R_{0}$. Hence, writing the balance between pressures with $\kappa^{-1}=R_{0}$ at threshold, we obtain a prediction for the gas speed $v_{c}$ above which bubbles form:

$$
v_{c}^{c-j}(\delta \simeq 0) \sim \sqrt{\frac{8 \gamma}{\rho_{g} R_{0}}} .
$$
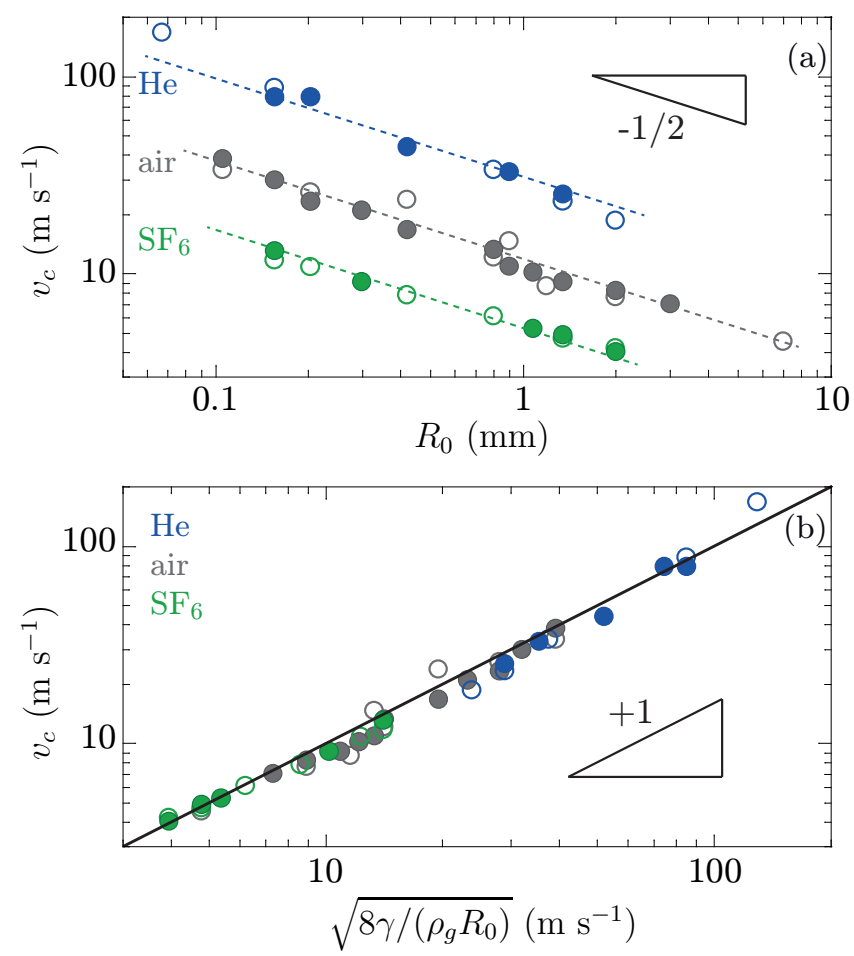

FIG. 2. (color online) (a) Variations for contained jets with $\delta \simeq 0$ of the velocity above which bubbles form $v_{c}$ with the nozzle radius $R_{0}$ for three different gases as indicated. The dashed lines are guides for the eyes having slopes $-1 / 2$. (b) Comparison between experiments shown in Fig. 2(a) and predictions given by Eq. (1) using 1 for the prefactor (solid line). Eq. (1) captures well experiments for both circular wands (open symbols) and vertically falling films (closed symbols). The film width is $w=50 \mathrm{~mm}$ so that $w /\left(2 R_{0}\right)=2-200$.

Our phenomenological model comparing gas inertia with surface tension allows us to obtain good quantitative agreement with experimental results as it collapses data shown in Fig. 2(a) onto a single curve and experi- 
ments are accurately described by choosing a value of 1 for the constant prefactor in Eq. (1) [see Fig. 2(b)].

To study the case of un-contained jets $[w / 2<R(\delta)]$ we use circular wands of diameter $w$ instead of our setup with vertically falling soap films. Indeed, the too large aspect ratios $h / w$ of the latter geometry prevent us from investigating un-contained jets with the setup shown in Fig. 1(b); in other words, in our experiments, $h \simeq 1 \mathrm{~m}$ is much larger than the largest value of $R_{0}$ which is $10 \mathrm{~cm}$.
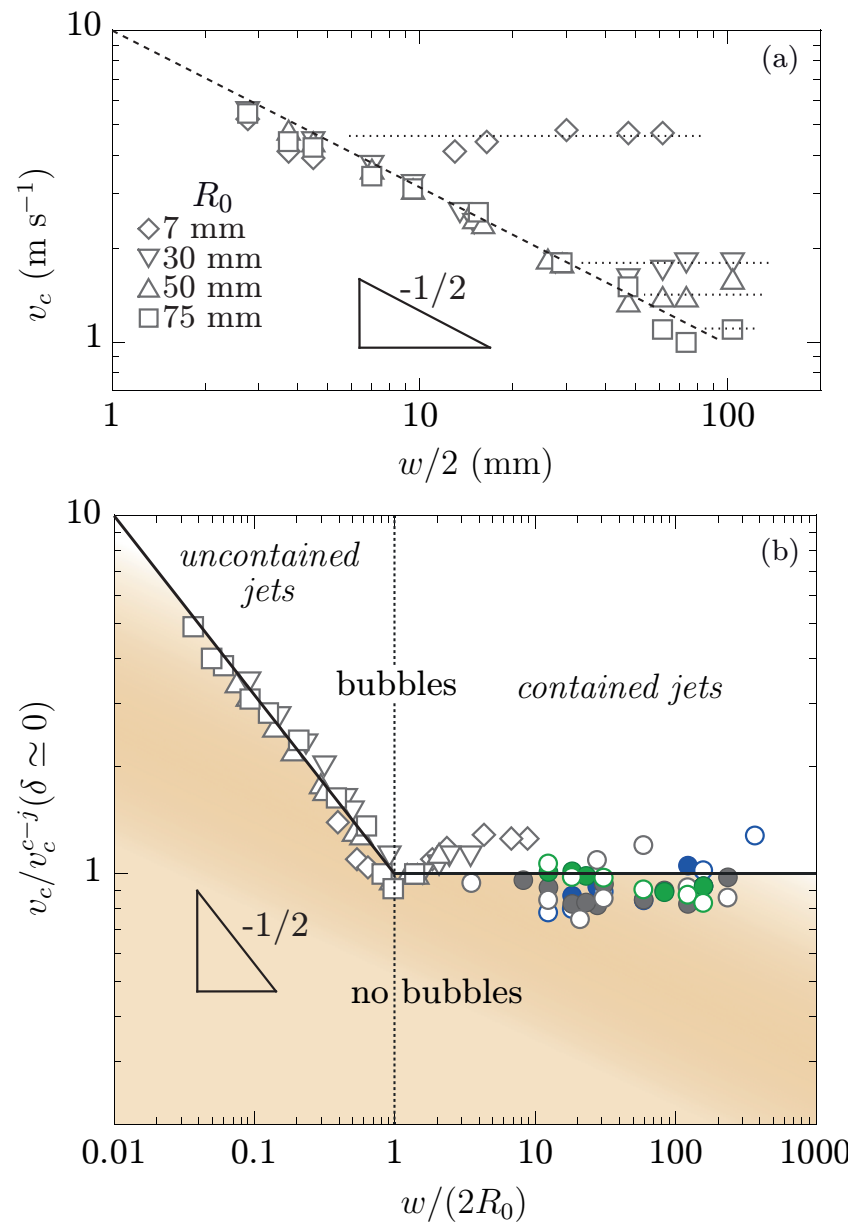

FIG. 3. (color online) (a) Evolution with $w / 2$ of the threshold velocity $v_{c}$ when $\delta \simeq 0$ for different nozzle radii as indicated. The degree of containment is $w /\left(2 R_{0}\right)=0.04-200$. The dashed and dotted lines are guides for the eyes having slopes $-1 / 2$ and 0 , respectively. (b) Dynamical characterization of the formation of bubbles when $\delta \simeq 0$ for jets that are either contained or un-contained. Solid lines correspond to the predictions for $v_{c} / v_{c}^{c-j}(\delta \simeq 0)$ given in the text with 1 for the prefactor. Data points correspond to those of Fig. 2 (all circles) and Fig. 3(a) (all triangles, diamonds, and squares).

We begin by studying the case $\delta \simeq 0$ for which $R(\delta)=R_{0}$. Similar to the contained configuration, observations at small enough gas speeds show that the jet deforms the soap film, creating a cavity whose radius of curvature $\kappa^{-1}$ decreases with $v_{g}<v_{c}$ and forming bub- bles at threshold when $v_{g}=v_{c}$. Hence, we can infer that the mechanism previously described that compares inertia and surface tension could also explain the variations of $v_{c}$ when $w / 2<R_{0}$. In this case, however, the size of the cavity is limited by the width of the frame which is smaller than the nozzle size so that the inertio-capillary mechanism discussed for $w / 2>R_{0}$ should now be written with $\kappa^{-1}=w / 2$ at threshold. The predicted gas velocity above which bubbles form in the un-contained case then reads $v_{c} \sim v_{c}^{c-j}(\delta \simeq 0) \sqrt{2 R_{0} / w}$. To validate this expression, we measure the variations of $v_{c}$ with $w / 2$ for different nozzle's radii. For a given nozzle and small enough $w / 2$, experiments confirm that $v_{c} \propto 1 / \sqrt{w}$ [see Fig. 3(a)]. Also shown in this figure, when $w / 2$ is much larger than $R_{0}$, the jets become contained and the experimental threshold velocity is no longer dependent on the soap film size in agreement with Eq. (1).

We summarize our findings for $\delta \simeq 0$ in Fig. 3(b) which, combining the results shown in Fig. 2 and Fig. 3(a), reports a diagram mapping the dynamics as functions of the dimensionless speed $v_{c} / v_{c}^{c-j}(\delta \simeq 0)$ and degree of containment $w /\left(2 R_{0}\right)$. As shown in this figure, our simple modeling work concurs well with experiments. As predicted by our physical arguments, the transitions between observed behaviors coincide with $w /\left(2 R_{0}\right)=1$ (un-contained vs contained jets), $v_{c} / v_{c}^{c-j}(\delta \simeq 0)=$ $\sqrt{2 R_{0} / w}$ (un-contained jets, bubbles vs no bubbles), and $v_{c} / v_{c}^{c-j}(\delta \simeq 0)=1$ (contained jets, bubbles vs no bubbles).

We now investigate the evolution of $v_{c}$ when the distance between nozzle's orifice and soap film is finite $(\delta \neq 0)$. Interestingly, this situation corresponds to the flow configuration encountered when blowing soap bubbles in the real world as illustrated in Fig. 1(a). In this case, predicting $v_{c}$ requires that our physical model comparing inertia and surface tension accounts for dissipation in the jet which is a function of $\delta$. To describe this realworld situation and to avoid possible buoyancy effects, in what follows we will work with air jets only. The shape and velocity structure of such turbulent jets entering a fluid at rest having the same density are known [21, 22]; see details in [19]. First, the jet adopts a nearly conical shape with an opening angle $\theta$ that depends neither on fluid properties, nor the jet speed, nor the size of the orifice, and is always approximately $23.6^{\circ}$ (see Fig. S2 in [19]). Hence, the jet radius at a distance $x$ from the orifice can be written $R(x)=R_{0}+x \tan \frac{\theta}{2} \simeq R_{0}+\frac{x}{5}$. Second, averaging over turbulent fluctuations, the velocity profile in the direction perpendicular to the $x$-axis exhibits a Gaussian shape; see Fig. S3 in [19] for measurements of such cross-jet velocity profiles.

In the contained configuration, using the shape and velocity structure of the jets discussed above, one easily shows that a generalized form of the balance between inertia and capillarity at threshold $\left[\kappa^{-1}=R(\delta)\right]$ reads 
$\frac{1}{2} \rho_{g} v_{c}^{2}\left(1+\frac{\delta}{5 R_{0}}\right)^{-1} \sim \frac{4 \gamma}{R_{0}}[19]$. Hence, the predicted gas velocity threshold is:

$$
v_{c} \sim \sqrt{\frac{8 \gamma}{\rho_{g} R_{0}}\left(1+\frac{\delta}{5 R_{0}}\right)} \sim v_{c}^{c-j}(\delta \simeq 0) \sqrt{1+\frac{\delta}{5 R_{0}}} .
$$

Similar to this analysis, in the case of un-contained jets for which $\kappa^{-1}=w / 2$ at threshold, one finds:

$$
v_{c} \sim v_{c}^{c-j}(\delta \simeq 0) \sqrt{\frac{2 R_{0}}{w}}\left(1+\frac{\delta}{5 R_{0}}\right) .
$$

When $\delta /\left(5 R_{0}\right)$ is much larger than 1, Eq.(2) and Eq.(3) predict that the variations of the threshold velocity $v_{c}$ with the distance $\delta$ should be weaker for contained jets $(\propto \sqrt{\delta})$ than for un-contained ones $(\propto \delta)$. These predictions concur very well with experiments (see Fig. 4). We summarize our predictions in Fig. 5.

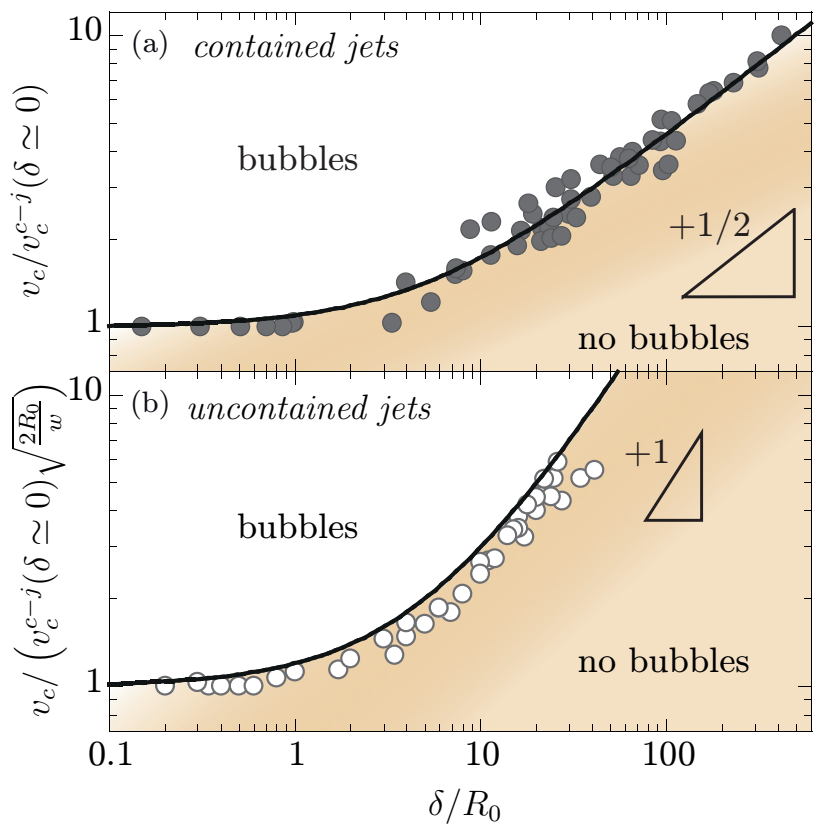

FIG. 4. (color online) Evolution with $\delta / R_{0}$ of the normalized threshold velocity for contained and un-contained jets. Solid lines stand for the predictions respectively given by Eq.(2) (contained jets) and Eq.(3) (un-contained jets) with 1 for the prefactor. Each data point stands for a value of the degree of containment: (a) $w /[2 R(\delta)]=10-500$ and (b) $w /[2 R(\delta)]=$ $0.13-0.87$

In closing, we have identified the physical factors governing the generation of soap bubbles when a gas jet impacts a soap film. To thoroughly study this hitherto uninvestigated simple experiment, we have built a bubble machine that mimics real-world bubble blowing and is based on vertically falling soap films. Using this machine, we have shown that bubbles form only when the gas speed

\begin{tabular}{|c|c|c|}
\hline$v_{c}$ & $\begin{array}{c}\text { contained jets } \\
R_{0}+\frac{\delta}{5}<\frac{w}{2}\end{array}$ & $\begin{array}{c}\text { uncontained jets } \\
R_{0}+\frac{\delta}{5}>\frac{w}{2}\end{array}$ \\
\hline$\delta \simeq 0$ & $v_{c}^{c-j}(\delta \simeq 0) \sim \sqrt{\frac{8 \gamma}{\rho_{g} R_{0}}}$ & $v_{c}^{c-j}(\delta \simeq 0) \sqrt{\frac{2 R_{0}}{w}}$ \\
\hline$\delta \neq 0$ & $v_{c}^{c-j}(\delta \simeq 0) \sqrt{1+\frac{\delta}{5 R_{0}}}$ & $v_{c}^{c-j}(\delta \simeq 0) \sqrt{\frac{2 R_{0}}{w}}\left(1+\frac{\delta}{5 R_{0}}\right)$ \\
\hline
\end{tabular}

FIG. 5. Table summarizing the predicted velocity threshold for making bubbles $v_{c}$ for each of the four flow configurations we have investigated.

exceeds a threshold that depends neither on the film's thickness nor hydrodynamics in our study. A competition between inertia and capillarity controls this velocity threshold which depends on containment, i.e. the ratio between soap film and jet size, and dissipation which is controlled by the distance from the soap film to the nozzle emitting the jet. The bubble machine will be particularly useful in generating long-lasting trains of bubbles to obtain production rate and bubble size statistics.

Acknowledgements: We thank l'Université Européenne de Bretagne and Rennes Métropole for support of this research (EPT Physfood), A. Saint-Jalmes for helping us with the surface tension and viscosity measurements, T. H. C.-Pancharatnam for blowing bubbles in Fig. 1(a), and E. Gicquel for lending us the wind tunnel.

* pascal.panizza@univ-rennes1.fr

laurent.courbin@univ-rennes1.fr

[1] I. B. Ivanov, Thin Liquid Films: Fundamentals and Applications (Surfactant Science Series, New York, 1988).

[2] F. Behroozi, Am. J. Phys. 76, 1087 (2008).

[3] J. Plateau, Statique Expérimentale et Théorique des Liquides Soumis aux Seules Forces Moléculaires (GauthierVillars, Paris, 1873).

[4] H. Bouasse, Capillarité-Phénomènes Superficiels (Librairie Delagrave, Paris, 1924).

[5] P. G. de Gennes, F. Brochard-Wyart, and D. Quéré, Capillarity and Wetting Phenomena: Drops, Bubbles, Pearls, Waves (Springer, New York, 2004).

[6] I. Cantat, S. Cohen-Addad, F. Elias, R. Höhler, O. Pitois, F. Rouyer, and A. Saint-Jalmes, Foams: Structure and Dynamics (Oxford University Press, Oxford, 2013).

[7] V. Cardoso and Ó. J. C. Dias, Phys. Rev. Lett. 96, 181601 (2006).

[8] K. Funakoshi, H. Suzuki, and S. Takeuchi, J. Am. Chem. Soc. 129, 12608 (2007).

[9] L. M. Custodio, Chest 114, 1224 (1998).

[10] J. Eggers and E. Villermaux, Rep. Prog. Phys. 71, 036601 (2008).

[11] M. A. Rutgers, X. L. Wu, and W. B. Daniel, Rev. Sci. Instrum. 72, 3025 (2001).

[12] M. A. Rutgers, X. L. Wu, R. Bhagavatula, A. A. Pe- 
tersen, and W. I. Goldburg, Phys. Fluids 8, 2847 (1996).

[13] H. Kellay and W. I. Goldburg, Rep. Prog. Phys. 65, 845 (2002).

[14] I. Kim and X. L. Wu, Phys. Rev. E 82, 026313 (2010).

[15] L. Courbin and H. A. Stone, Phys. Fluids 18, 091105 (2006).

[16] A. Le Goff, L. Courbin, H. A. Stone, and D. Quéré, EPL 84, 36001 (2008).

[17] T. Gilet and J. W. M. Bush, J. Fluid Mech. 625, 167 (2009).

[18] G. Kirstetter, C. Raufaste, and F. Celestini, Phys. Rev. E 86, 036303 (2012).
[19] See Supplemental Material at [] for a brief discussion about the role of the thickness and hydrodynamics of films on bubble blowing, details about the shape and velocity structure of the gas jets used in this study, and two movies illustrating the generation of cavities and soap bubbles in vertical films.

[20] We consider that $\delta \simeq 0$ when $\delta \ll R_{0}$ and $\delta \ll w$.

[21] S. B. Pope, Turbulent Flows (Cambridge University Press, Cambridge, 2000).

[22] G. Horn and M. W. Thring, Nature 178, 205 (1956). 